\title{
Actualización en el manejo de pacientes con insuficiencia intestinal
}

\author{
GIGLIOLA ALBERTI R. ${ }^{1}$, CATALINA LE ROY O. ${ }^{1}$, COLOMBA COFRÉ D. ${ }^{1}$, \\ JUAN CARLOS PATTILLO S. ${ }^{2}$, PILAR DOMÍNGUEZ B. ${ }^{3}$, JUAN FRANCISCO GUERRA C. ${ }^{3}$
}

1. Departamento de Gastroenterología y Nutrición, División de Pediatría, Escuela de Medicina, Pontificia Universidad Católica de Chile.

2. Sección de Cirugía Pediátrica, División de Cirugía, Escuela de Medicina, Pontificia Universidad Católica de Chile.

3. Departamento de Cirugía Digestiva, División de Cirugía, Escuela de Medicina, Pontificia Universidad Católica de Chile.

\begin{abstract}
Advances in the management of children with intestinal failure

Intestinal failure is defined as the reduction of a functional gut mass below the minimal necessary for adequate digestion and absorption of nutrients and fluids. Intestinal failure is the final result of a number of different causes, being short bowel syndrome the most recognized. Its prevalence is low, but the impact in quality of life among patients and their families is critical. Furthermore, is associated with high economic costs, both for the patient and the health provider. Its main feature is the need for long-term parenteral nutritional support with high morbidity and mortality associated complications, such as line-derived bloodstream infections and liver disease. The management of these patients should be performed by a multidisciplinary team, and be aimed at promoting adaptation and recovery of bowel function to achieve intestinal autonomy.

(Key words: Intestinal failure, short bowel syndrome, small bowel transplantation).

Rev Chil Pediatr 2014; 85 (2): 148-156
\end{abstract}

\section{RESUMEN}

La insuficiencia intestinal se define como la reducción de la masa intestinal funcional por debajo de la cantidad mínima necesaria para la digestión y la absorción adecuada de nutrientes y fluidos. Responde a diferentes causas, siendo la más reconocida el intestino corto. En este trabajo se revisarán los recientes avances en el manejo médico y quirúrgico de esta entidad, que, siendo una patología de baja prevalencia, afecta directamente la calidad de vida de los pacientes y sus familias, y se asocia a altos costos económicos. Su principal característica es la necesidad de soporte nutricional a largo plazo, y sus mayores complicaciones son sepsis y enfermedad hepática secundaria al uso prolongado de nutrición parenteral. El manejo de estos pacientes debe ser realizado por un equipo multidisciplinario, y estar orientado a promover la adaptación y recuperación de la función del intestino para alcanzar su autonomía intestinal. Su derivación temprana a un centro especializado está fuer-

Recibido el 18 de marzo de 2014, aceptado para publicación el 9 de abril de 2014.

Este trabajo cumple con los requisitos sobre consentimiento /asentimiento informado, comité de ética, financiamiento, estudios animales y sobre la ausencia de conflictos de intereses según corresponda.

Correspondencia a:

Dra. Gigliola Alberti Reus

E-mail: galberti@med.puc.cl 
temente recomendada, ojalá antes de la aparición de complicaciones. El trasplante intestinal, incipiente en nuestro medio, es una alternativa terapéutica en casos seleccionados, en los que la rehabilitación intestinal no ha sido alcanzada

(Palabras clave: Insuficiencia intestinal, intestino corto, trasplante intestinal).

Rev Chil Pediatr 2014; 85 (2): 148-156

\section{Introducción}

La insuficiencia intestinal (II) es una enfermedad poco frecuente pero constituye un evento de alto impacto para el niño y su familia, y requiere de una atención especializada y multidisciplinaria. Se define como la incapacidad de alcanzar un adecuado balance proteico, energético, hídrico, electrolítico y de micronutrientes producto de una reducción de la masa funcional enterocitaria que permita una adecuada digestión y absorción ${ }^{1}$. Puede producirse por diferentes causas (tabla 1), como anatómicas, en las que existe un compromiso de la longitud intestinal producto de malformaciones congénitas o por resecciones quirúrgicas, $\mathrm{y}$ funcionales, como dismotilidad intestinal y enfermedades asociadas a malabsorción. La característica común a estos pacientes es la necesidad de un soporte nutricional prolongado, con requerimientos de Nutrición Parenteral (NP) variables en el tiempo, aunque habitualmente bastante extensos. El manejo de estos niños es complejo, necesita de personal de las distintas especialidades, altamente calificado y que idealmente forme parte de un programa

Tabla 1. Principales causas de Insuficiencia Intestinal

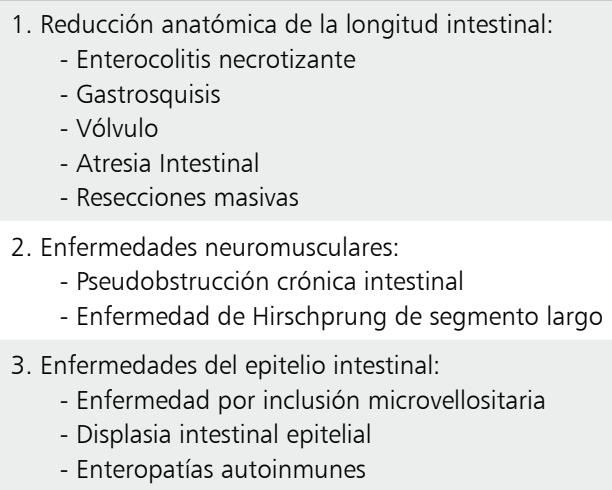

2. Enfermedades neuromusculares:

- Pseudobstrucción crónica intestinal

- Enfermedad de Hirschprung de segmento largo

3. Enfermedades del epitelio intestinal: Enfermedad por inclusión microvellositaria - Displasia intestinal epitelial - Enteropatías autoinmunes

formal de Rehabilitación intestinal, para así alcanzar el objetivo principal en estos pacientes: lograr su autonomía enteral.

\section{Adaptación intestinal}

La adaptación intestinal es un proceso que tiene como objetivo restaurar la absorción normal de macronutrientes, agua y minerales ${ }^{2}$. Tiene un componente estructural, con un incremento del área absortiva del intestino remanente caracterizado por profundización de las criptas, con hiperplasia de sus células y crecimiento en longitud de las vellosidades intestinales, y otro componente funcional, caracterizado principalmente por el enlentecimiento del tránsito gastrointestinal. Estos cambios adaptativos comienzan precozmente, dentro de las 12 a 24 primeras horas luego de la resección intestinal y se mantienen por varios meses o incluso años ${ }^{3}$.

Existe una serie de factores que han sido reconocidos como pronósticos en el proceso de adaptación intestinal, como son el uso de la vía enteral, mayor longitud del intestino delgado, condición del intestino residual, presencia de válvula ileocecal, ausencia de sobrecrecimiento bacteriano, presencia de colon y de continuidad intestinal ${ }^{4,5}$.

En los últimos años, se ha trabajado en la búsqueda de biomarcadores no invasivos que sean capaces de traducir la longitud y/o el funcionamiento del intestino remanente y la capacidad de adaptación en pacientes con intestino corto. La citrulina es un aminoácido no esencial sintetizado exclusivamente en el enterocito, que es liberado a la circulación sistémica y convertido en Arginina en las células del riñón. Se ha encontrado relación entre las concentraciones plasmáticas de citrulina y la longitud intestinal remanente en intestinos cortos anatómicos (masa enterocítica), la capacidad 
absortiva del intestino delgado (adaptación intestinal) y la probabilidad de retiro de NP. Es así como los niveles de citrulina se consideran un factor pronóstico en retiro de NP. Las distintas publicaciones muestran que niños con valores de citrulina $>15-20 \mu \mathrm{mol} / \mathrm{L}$ logran ser retirados de NP, en cambio niños con valores de citrulina $<11-12 \mu \mathrm{mol} / \mathrm{L}$ no logran independizarse de ella ${ }^{6-8}$.

\section{Rehabilitación intestinal}

El objetivo primero y hacia donde debe estar orientado el manejo de estos niños es lograr su rehabilitación intestinal. Para ello, es fundamental el trabajo multidisciplinario, de un grupo humano que incluya nutriólogos, gastroenterólogos, infectólogos, cirujanos y enfermeras, abarcando así los diferentes problemas que afectan a dichos pacientes. Existe evidencia que demuestra que el manejo multidisciplinario de los pacientes con II, asociado a un programa formal de rehabilitación intestinal, mejora drásticamente su pronóstico ${ }^{9}$.

\section{Manejo nutricional}

El soporte nutricional es un componente crítico en el cuidado de los pacientes con II, y tiene que ir enfocado en lograr un crecimiento y desarrollo lo más adecuado posible, tratando siempre de evitar las principales complicaciones que afectan a estos niños.

\section{Nutrición enteral}

La nutrición enteral es primordial para una adaptación intestinal exitosa, ya que promueve la hiperplasia de la mucosa y mejora la absorción por contacto directo con los nutrientes y a través de la liberación de factores humorales ${ }^{10}$. En pacientes que no presenten contraindicaciones, la nutrición enteral debe ser iniciada lo antes posible. La elección de los nutrientes va a depender de la capacidad de digestión y absorción del intestino remanente. La primera alternativa es la leche materna. Si no se dispone de ella, se pueden utilizar fórmulas comerciales, idealmente con baja osmolaridad y sustratos rápidamente absorbibles, que no dejen residuos que puedan producir diarrea osmótica.
Estudios observacionales han identificado que factores como la introducción precoz de la alimentación enteral, la ingesta de leche materna $\mathrm{y}$ el uso de fórmulas aminoacídicas permiten el retiro de NP más tempranamente ${ }^{11,12}$.

\section{Nutrición parenteral}

La NP es el pilar principal en la terapia de estos pacientes, y su objetivo es compensar las pérdidas intestinales de fluidos y entregar el aporte nutricional para un crecimiento óptimo. Su uso ha permitido prolongar la vida de muchos niños antiguamente considerados "fuera de alcance terapéutico", pero en el largo plazo puede producir complicaciones serias, siendo las principales las infecciones asociadas a catéter venoso central (CVC) y la falla hepática asociada a NP (FHANP). La prescripción de la NP debe ser individualizada, siempre tratando de alcanzar la mínima dependencia posible. El uso exclusivo o total de NP debe ser evitado, ya que un alto grado de dependencia de NP es un factor de riesgo para el desarrollo de complicaciones metabólicas ${ }^{13}$.

\section{Sobrecrecimiento bacteriano}

El sobrecrecimiento bacteriano (SB) es una complicación frecuente de los pacientes con II, y puede influir en el proceso de adaptación intestinal, por lo que es importante saber reconocerlo y manejarlo. Se define como la proliferación de flora bacteriana de tipo colónico en el intestino delgado que produce alteración de la digestión y absorción de nutrientes ${ }^{14}$. Las especies más frecuentemente encontradas son Streptococcus, Escherichia coli, Bacteroides y Lactobacillus ${ }^{15}$. Dentro de los factores predisponentes para su desarrollo destacan los factores anatómicos, como resección de la válvula ileocecal y la presencia de asas ciegas y estenosis, factores funcionales, como dilatación y enlentecimiento intestinales, y uso de medicamentos como bloqueadores del ácido y antidiarreicos (por ejemplo: Loperamida).

El SB tiene consecuencias deletéreas sobre la digestión y absorción de nutrientes y produce cambios inflamatorios en la mucosa intestinal con el consiguiente aumento de la permeabilidad y eventual riesgo de translocación 
bacteriana y sepsis. Se ha descrito también un mayor riesgo de desarrollo de esteatosis hepática y exacerbación de la hepatotoxicidad ligada a nutrición parenteral ${ }^{16}$. El diagnóstico puede hacerse de forma indirecta mediante el test de hidrógeno en aire espirado posterior a una sobrecarga oral de lactulosa, o bien de forma directa por aspiración y cultivo de contenido intestinal obtenido endoscópicamente. Su tratamiento consiste en el uso de antibióticos empíricos, cubriendo bacterias aerobias y anaerobias. Debido a que las causas de SB en estos pacientes se mantienen sin modificaciones a lo largo del tiempo, muchas veces es necesario usar antibióticos de manera repetida, en ciclos y alternando distintos tipos para disminuir el desarrollo de resistencia bacteriana ${ }^{17}$.

\section{Avances en el manejo de la II}

\section{Uso de emulsiones lipídicas en base a aceite de pescado}

La FHANP es una de las principales y más devastadoras complicaciones de los pacientes usuarios de NP prolongada, y su principal manifestación es la colestasia. Aunque su patogenia es multifactorial, una serie de estudios ha implicado tanto a la cantidad como al tipo de lípidos de uso parenteral en su desarro$10^{18}$. Las emulsiones lipídicas habitualmente usadas, que están basadas en aceite de soya, contienen ácidos grasos Omega 6, con acción proinflamatoria, los que sumados a los fitoesteroles, pueden contribuir a la hepatoxicidad. En contraste, las formulaciones lipídicas en base a aceite de pescado son ricas en ácidos grasos Omega 3, con acción antinflamatoria y protectora del hígado ${ }^{19}$. Actualmente existen en el mercado formulaciones lipídicas que contienen $100 \%$ aceite de pescado (Omegaven $\AA$ ) y otras con una mezcla de éste y aceites de soya, coco y oliva (SMOF®). La evidencia muestra que estas emulsiones lipídicas en base a aceite de pescado, en contraste a las emulsiones habituales, podrían prevenir y revertir la FHANP.

\section{Lock terapia con Etanol}

En niños con II, el uso de CVC es indispensable, pero las complicaciones derivadas de su uso, siendo la principal la infección asociada a CVC, contribuyen significativamente a la morbilidad de los pacientes y a aumentar los costos de atención ${ }^{20}$. Dependiendo de la serie, las tasas de infecciones relacionadas con CVC en niños con II van desde 8 hasta 14 infecciones por cada 1.000 días de catéter. Es de vital importancia un buen manejo de enfermería en la prevención de dichas infecciones, y recordar que muchas de ellas pueden ser tratadas sin la necesidad de retirar el dispositivo. El etanol tiene una acción bactericida y fungicida, y su uso como lock terapia en concentración $70 \%$ es una estrategia eficaz para la prevención de infecciones asociadas a CVC. Existen diferentes esquemas de uso, que van desde la instilación por entre 2 a $4 \mathrm{~h}$ al día, ya sea diariamente o entre una a tres veces a la semana, todos con resultados similares. Con esta terapia, las infecciones asociadas a CVC pueden disminuir entre 0,9 y 2,8 infecciones por cada 1.000 días de catéter ${ }^{21}$, por lo que su uso está fuertemente recomendado.

\section{Teduglutide}

Es un análago de glucagon-like peptide 2 (GLP-2), una hormona de vida media muy corta, secretada normalmente por las células L del íleon terminal y colon ${ }^{22}$. Es la primera terapia de largo plazo aprobada en EEUU para el tratamiento de pacientes adultos con síndrome de intestino corto que dependen de NP. Aumenta la altura vellositaria y la profundidad de las criptas del epitelio intestinal resultando en una mejoría de la capacidad absortiva, facilita la reducción en el soporte de volumen parenteral y otorga días libres de NP en pacientes con SIC $^{23}$. Hasta ahora, el uso de Teduglutide está estudiado sólo en adultos, por lo que su uso no se puede recomendar en niños.

\section{Manejo quirúrgico inicial de la II}

El manejo multidisciplinario de los pacientes con II demanda una activa participación de cirujanos pediátricos en la toma de decisiones que serán trascendentales en el pronóstico y la potencialidad de rehabilitación enteral de este grupo de niños ${ }^{24}$. Su rol comienza con la educación y difusión médica de patologías asociadas a II que se beneficiarán de una derivación 
oportuna. Desde el período antenatal es posible diagnosticar o sospechar patologías como gastrosquisis o atresia intestinal (figura 1). En recién nacidos con vómitos biliosos siempre debe sospecharse la presencia de vólvulo de intestino medio, el que deberá ser descartado con un estudio contrastado de esófago, estómago y duodeno ${ }^{25}$. Nunca se debe olvidar a los pacientes con enterocolitis necrotizante, que pudiesen requerir resecciones intestinales masivas.

Se deben identificar precozmente aquellos pacientes en riesgo de II, considerando en ellos alternativas de tratamiento que permitan conservar la mayor longitud de intestino posible. Cuando la injuria isquémica está establecida y el intestino se observa necrótico y sin viabilidad, la resección quirúrgica está indicada. Sin embargo, debe ser lo más acotada posible, cuidando de conservar intestino de viabilidad marginal susceptible de recuperarse.

\section{Accesos enterales}

Los accesos enterales favorecen la rehabilitación intestinal y permiten una adecuada titulación de los aportes. La utilización de gastrostomías (laparoscópicas, abiertas o percutáneas) está indicada cuando existe necesidad de acceso enteral de largo plazo. La utilización de tubos o botones gastroyeyunales permiten drenar el estómago en caso de dismotilidad gástrica manteniendo la posibilidad de realizar aporte con fórmulas enterales.

\section{Accesos vasculares}

La utilización de accesos venosos para NP y la administración de medicamentos suele plantearse tempranamente en pacientes con II y debe ser planificada considerando su potencial uso por tiempo prolongado. Los catéteres utilizados pueden ser tunelizados con cuff (Hickman o Broviack) o sin cuff e insertados a través de venas periféricas (peripherally inserted central catheter o "PICC"). Independiente de la técnica utilizada, punción o denudación, la recomendación es comenzar por afluentes venosos periféricos manteniendo la indemnidad de las venas centrales, sobretodo evitando ligarlas. Es una buena práctica, incluso en recién nacidos pequeños, conservar venas

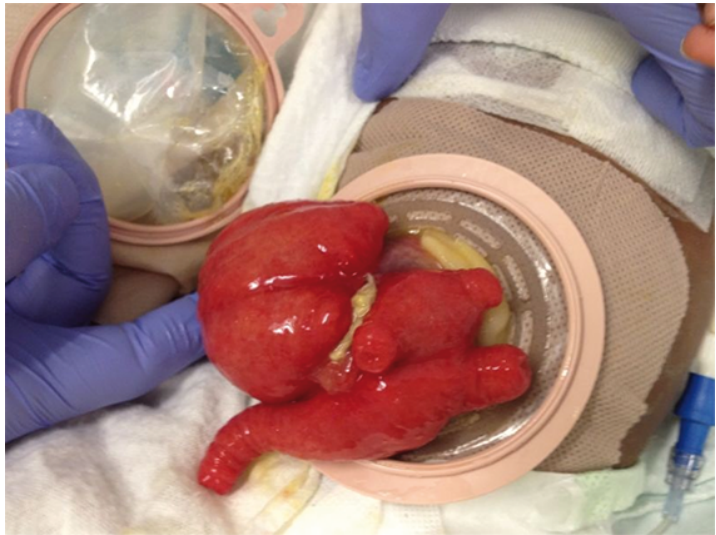

Figura 1. Paciente con gastrosquisis y múltiples fístulas.

como la yugular externa o safena mayor para la inserción de líneas centrales. La instalación de estos dispositivos debe ser realizada por equipos de cirujanos con experiencia y con visión de largo plazo en el manejo de niños con II. El desarrollo de técnicas quirúrgicas y de radiología intervencional ha permitido el uso de recursos venosos impensados en el pasado, como las venas ácigos, gonadales, suprahepáticas o lumbares. Sin embargo, el capital venoso humano es limitado, y la pérdida de accesos venosos es una indicación aceptada de trasplante intestinal, dando cuenta de cerca del $50 \%$ de sus derivaciones ${ }^{26}$.

\section{Cirugías de rehabilitación intestinal}

Desde comienzos de los años ochenta ha habido intentos por desarrollar técnicas quirúrgicas de rehabilitación intestinal, pero la gran mayoría de ellas han sido abandonadas por no presentar beneficios a largo plazo. Su principal objetivo es maximizar la función residual de manera simple. Hoy en día existen dos técnicas de reconstrucción utilizadas preferentemente: la técnica de alargamiento longitudinal de Bianchi y el STEP (serial transverse enteroplasty).

Las técnicas quirúrgicas de rehabilitación intestinal se ocupan de cuatro aspectos fisiopatológicos de la insuficiencia intestinal ${ }^{27}$ :

1. Optimización de intestino residual a través del cierre de ostomías, manejo adecuado de fístulas éntero-cutáneas, liberación de 
estenosis y obstrucciones, y de asas ciegas.

2. Mejoría de la motilidad intestinal en asas dilatadas o con dismotilidad con técnicas de plicatura o inversión.

3. Enlentecimiento del tránsito acelerado utilizando asas reversas, válvulas semiobstructivas o colon.

4. Reconstrucción autóloga (Bianchi, STEP o ambas).

Cuando todos los métodos para lograr el máximo de intestino se han utilizado, entonces debe considerarse una técnica de alargamiento. Esto ocurre habitualmente después del primer año de vida. La técnica descrita por Bianchi en 1980 fue la primera en intentar una reconstrucción autóloga del intestino y consiste básicamente en dividir el intestino dilatado en dos mitades, preservando irrigación independiente en cada una. De esta forma se obtienen dos tubos de la mitad del diámetro del original. Éstos se anastomosan con técnica término terminal, duplicando la longitud inicial del segmento.

La técnica STEP fue descrita por Kim en 2003. En esta técnica el intestino dilatado se aplana juntando las caras mesentéricas y antimesentéricas para luego seccionar con stapler en forma perpendicular al mesenterio y alternadamente a cada lado de éste en forma equidistante, creando un zig-zag de longitud superior al tubo original.

Quedan algunas interrogantes por responder: ¿cuál es el mejor momento para realizar estas operaciones?, ¿qué pacientes se beneficiarán de una u otra técnica? A través del análisis sistemático de casos y series publicados en la literatura se encuentra información valiosa: no parece haber diferencia significativa en la sobrevida de los pacientes sometidos a uno $\mathrm{u}$ otro procedimiento, los pacientes sometidos a elongación intestinal según Bianchi tienen menos complicaciones y un mayor porcentaje de ellos puede prescindir de NP, mientras que el enlistamiento para trasplante intestinal es proporcionalmente menor en pacientes sometidos a STEP. Estos datos deben ser analizados con cautela, pues podrían estar sesgados por los tiempos de seguimiento, especialmente considerando que el STEP tiene 10 años de existencia ${ }^{28}$.
Los avances en el manejo médico, el mejor cuidado de accesos venosos y la cirugía de rehabilitación intestinal, han permitido que cada vez menos pacientes con intestino corto como causa de II requieran trasplantes viscerales ${ }^{29,30}$.

\section{Trasplante intestinal}

\section{Generalidades}

El trasplante intestinal (TI) está indicado para pacientes en los cuales la rehabilitación no es posible o ésta ha fracasado ${ }^{31}$. Los primeros intentos de TI fueron realizados hace ya casi un siglo por Alexis Carrell, pero ha sido durante los últimos 30 años cuando debido a la mejoría de las técnicas quirúrgicas, cuidados perioperatorios y muy especialmente la racionalización de la inmunosupresión, se ha logrado obtener resultados sostenidamente mejores.

\section{Indicaciones}

Las principales indicaciones de TI son aquellas derivadas del uso permanente de NP, así como del CVC: trombosis venosas, episodios de infecciones graves, admisiones frecuentes por deshidratación o desequilibrios hidroelectrolíticos y la insuficiencia hepática asociada a NP ${ }^{31-33}$ (tabla 2).

\section{Tipos de trasplante intestinal}

El TI debe ser individualizado para cada paciente, basado en su indicación y la anatomía y fisiología del candidato ${ }^{31,34}$. Existen tres tipos de procedimientos quirúrgicos:

1. Trasplante intestinal aislado: El trasplante incluye solamente el intestino delgado (o alguna porción del colon). Corresponde al tipo de trasplante más frecuente en adultos y niños mayores.

\section{Tabla 2. Indicaciones de Trasplante intestinal ${ }^{40}$}

1) Falla hepática inminente o evidente

2) Trombosis de dos o más venas centrales

3) Dos o más episodios de sepsis asociadas a CVC por año

4) Un episodio de infección fúngica asociada a CVC, shock séptico o distress respiratorio agudo

5) Episodios frecuentes de deshidratación severa a pesar de la suplementación con volumen adicional a la NP 
2. Trasplante higado-intestino: Fue desarrollado inicialmente para pacientes pediátricos pero su uso se ha masificado con la mayor cantidad de pacientes portadores de FHANP. El injerto está compuesto por el hígado (o una porción de éste) más un segmento de intestino delgado de longitud variable y el complejo duodeno-pancreatobiliar, disminuyendo así las potenciales complicaciones derivadas de una anastomosis biliar (figura 2).

3. Trasplante multivisceral: Debe su nombre a la presencia del estómago en el injerto, junto a los órganos antes descritos para el trasplante hígado-intestino. Su indicación principal la constituyen las trombosis mesentéricas extensas o diversas condiciones que impliquen un estómago no funcionante o ausente.

\section{Complicaciones}

La formación continua de los diversos profesionales relacionados con el trasplante así como mejores cuidados peri y postoperatorios han significado una mejoría progresiva de los resultados. Aún así, sus principales complicaciones son:

Quirúrgicas: La naturaleza y la complejidad del procedimiento quirúrgico hacen que trombosis vasculares, hemorragias, perforaciones y complicaciones derivadas de ostomías sean comunes, aunque hoy en día son menos frecuentes como causa de pérdidas de injertos o pacientes.

Inmunológicas: Debido a la elevada carga inmunológica del intestino, este tipo de trasplantes requiere de inmunosupresión potente. Conocida es la secuencia rechazo grave, disfunción del injerto y potencial muerte del receptor, por lo tanto debe ponerse especial énfasis en la vigilancia inmunológica mediante endoscopías seriadas que detectarán un rechazo en etapas tempranas y permitirán la realización de modificaciones en la terapia inmunosupresora. Diversos esquemas de inducción han disminuido sustancialmente la incidencia de rechazo ${ }^{35,36}$.

Infecciosas: Las infecciones post TI son comunes y están en estrecha relación con la magnitud de la inmunosupresión utilizada ${ }^{37}$.

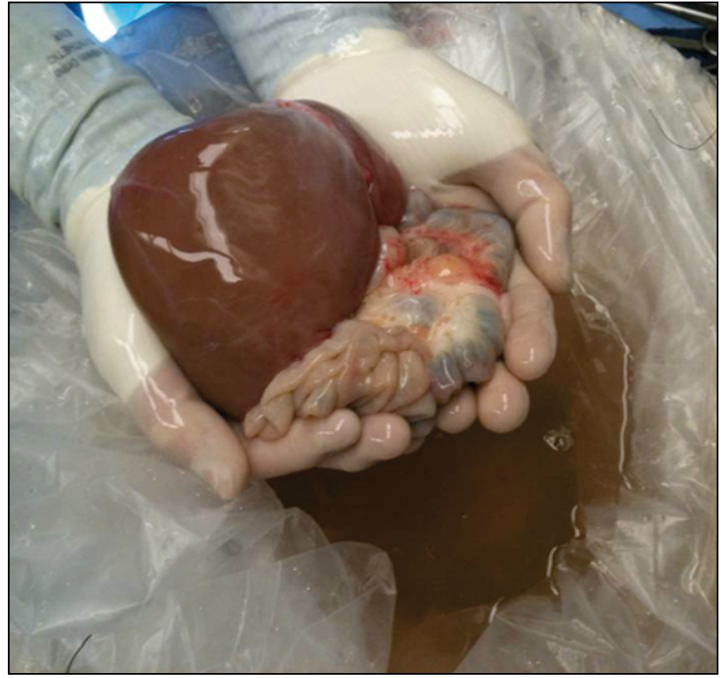

Figura 2. Injerto pediátrico Hígado-Intestino.

Usualmente, las infecciones siguen temporalmente a un curso de tratamiento por rechazo en un paciente severamente inmunosuprimido. Infecciones virales, bacterianas y fúngicas pueden atentar contra el injerto y la vida del paciente. Nuevamente, la vigilancia estricta resulta fundamental como estrategia para disminuir la incidencia e impacto de las infecciones sobre el receptor.

\section{Resultados}

En centros especializados, las sobrevidas de pacientes a 1 y 5 años alcanzan $85-90 \%$ y $60-70 \%$ respectivamente ${ }^{31,38}$. Desde el punto de vista nutricional, un $90 \%$ de los pacientes son capaces de ser liberados de NP durante el primer año. Es importante destacar que en el análisis de los resultados, cada vez parece ser más relevante el estado del paciente antes del trasplante, de ahí la importancia de la comunicación temprana entre el equipo multidisciplinario de insuficiencia y trasplante intestinal y el centro derivador.

Como conclusión, podemos señalar que el progreso sostenido del TI hace de esta una técnica estándar para pacientes sin posibilidad de rehabilitación intestinal. Resulta fundamental la estricta selección de los candidatos, la integración temprana y fluida entre el centro derivador y el centro trasplantador y la realización 


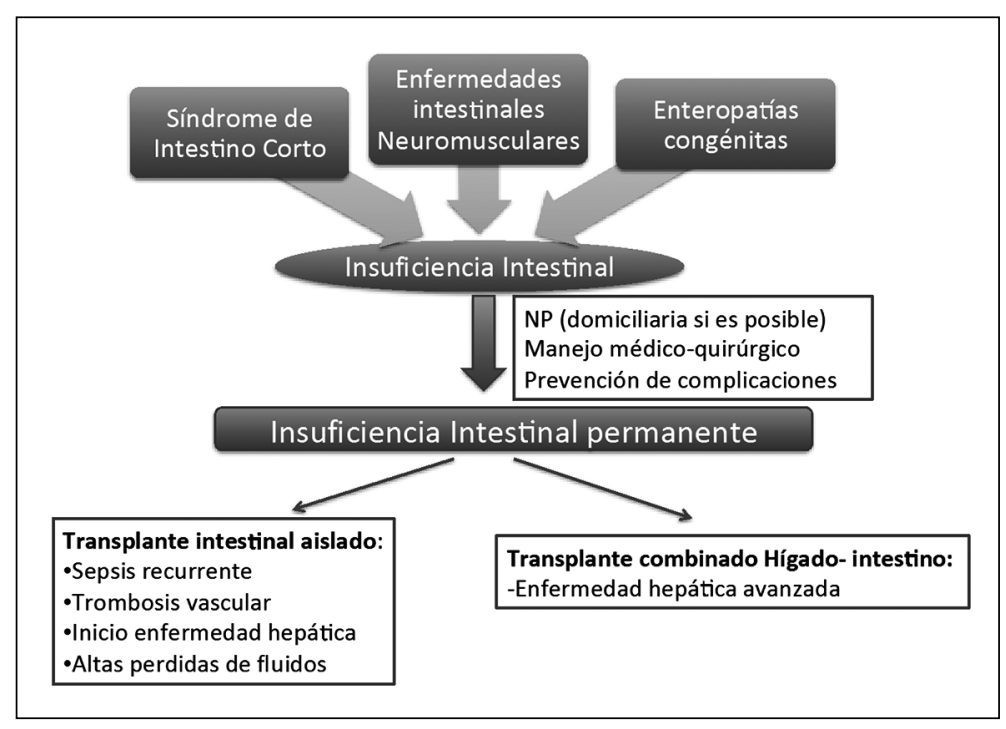

Figura 3. Resumen del manejo de pacientes con Insuficiencia Intestinal. de casos en centros con protocolos y personas especializadas. Dada la importancia de la variable inmunológica así como la incidencia del rechazo, se están destinando importantes esfuerzos ${ }^{39}$ para el desarrollo de biomarcadores que permitan una evaluación más temprana del funcionamiento del injerto.

\section{Conclusiones}

El manejo de pacientes con insuficiencia intestinal es complejo y debe realizarse por un equipo multidisciplinario. Representa un desafío para los diferentes especialistas involucrados, quienes deben orientar siempre su trabajo hacia el logro de la rehabilitación intestinal y la autonomía enteral. La derivación temprana de dichos pacientes a un centro especializado está fuertemente recomendada, idealmente antes de la aparición de complicaciones (figura 3).

\section{Referencias}

1.- O'Keefe SJ, Buchman AL, Fishbein TM, et al: Short bowel syndrome and intestinal failure: consensus definitions and overview. Clin Gastroenterol Hepatol 2006; 4: 6-10.

2.- Goodlad RA, Nightingale JMD, Playford RJ: Intestinal adaptation. In: Nightingale JMD, eds. Intestinal failure. Greenwich: Greenwich Medical Media Limited, 2001;
243-60.

3.- American Gastroenterological Association: Medical position statement: short bowel syndrome and intestinal transplantation. Gastroenterology 2003; 124: 1105-10.

4.- Quiros-Tejeira RE, Ament ME, Reyen L, et al: Longterm parenteral nutrition support and intestinal adaptation in children with short bowel syndrome: a 25 year experience. J Pediatr 2004; 145: 157-63.

5.- Warner B: Adaptation: Paradigm for the gut and a academic career. J Ped Surg 2013; 48: 20-6.

6.- Bailly-Botuha C, Colomb V, Thioulouse E, et al: Plasma citrulline concentration reflects enterocyte mass in children with short bowel syndrome. Pediatr Res 2009; 65: 559-63.

7.- Fitzgibbons $S$, Ching YA, Valim C, et al: Relationship between serum citrulline levels and progression to parenteral nutrition independence in children with short bowel syndrome. J Pediatr Surg 2009; 44: 928-32.

8.- Stultz JS, Tillman EM, Helms RA: Plasma citrulline concentration as a biomarker for bowel loss and adaptation in hospitalized pediatric patients requiring parenteral nutrition. Nutr Clin Pract 2011; 26: 681-7.

9.- Torres C, Sudan D, Vanderhoof J, et al: Role of an Intestinal Rehabilitation Program in the Treatment of Advanced Intestinal Failure. J Pediatr Gastroenterol Nutr 2007; 45 (2).

10.- Martin GR, Wallace LE, Hartmann B, et al: Nutrientstimulated GLP-2 release and crypt cell proliferation in experimental short bowel syndrome. Am J Physiol Gastrointestinal Liver Physiol 2005; 288: G431-8.

11.- Bines J, Francis D, Hill D: Reducing parenteral require- 
ment in children with short bowel syndrome: impact of an amino acid based complete infant formula. J Pediatr Gastroenterol Nutr 1998; 26 (2): 123-8.

12.- Andorsky DJ, Lund DP, Lillehei CW, et al: Nutritional and other postoperative management of neonates with short bowel syndrome correlates with clinical outcomes. J Pediatr 2001; 139: 27-33.

13.- Kaufman SS: Prevention of PNALD in children. Pediatr Transplantation 2002; 6: 37-42.

14.- Ballesteros $M D$, Vidal A: Síndrome de intestino corto: definición, causas, adaptación intestinal y sobrecrecimiento bacteriano. Nutr Hosp 2007; 22: 74-85.

15.- Bouhnik Y, Alain S, Attar A, et al: Bacterial populations contaminating the upper gut in patients with small intestinal bacterial overgrowth syndrome. Am J Gastroenterol 1999; 94: 1327-31.

16.- Wolf A, Pohlandt F: Bacterial infection: the main cause of acute cholestasis in newborn infants receiving shortterm PN. J Pediatr Gastroenterol Nutr 1989; 8: 297-303.

17.- Dibaise JK, Young RJ, Vanderhoof JA: Enteric microbial flora, bacterial overgrowth, and short-bowel syndrome. Clin Gastroenterol Hepatol 2006; 4: 11-20.

18.- Zi-Wei Xu, You-Sheng Li: Pathogenesis and treatment of parenteral nutrition-associated liver disease. Hepatobiliary Pancreat Dis Int 2012; 11: 586-93.

19.- Fallon EM, Le HD, Puder M: Prevention of parenteral nutrition- associated liver disease: role of omega-3 fish oil. Curr Opin Organ Transplant 2010; 15 (3): 334-40.

20.- Flynn PM: Diagnosis and management of central venous catheter-related bloodstream infections in pediatric patients. Pediatr Infect Dis J 2009; 28: 1016-7.

21.- Peterson J, Kerner J: New Advances in the Management of Children With Intestinal Failure. J Parenter Enteral Nutr 2012; 36: 36S-42S.

22.- Jeppesen PB, Sanguinetti EL, Buchman A, et al: Teduglutide (ALX-0600), a dipeptidyl peptidase IV resistant glucagon-like peptide 2 analogue, improves intestinal function in short bowel syndrome patients. Gut 2005; 54 (9): 1224-31.

23.- Jeppesen PB, Pertkiewicz M, Messing B, et al: Teduglutide Reduces Need for Parenteral Support Among Patients With Short Bowel Syndrome With Intestinal Failure. Gastroenterology 2012; 143: 1473-81.

24.- Biren P. Modi, MD, Tom Jaksic: Pediatric Intestinal Failure and Vascular Access. Surg Clin N Am 2012; 92 : 729-43.

25.- Williams $H$ : Green for danger! Intestinal malrotation and volvulus. Arch Dis Child Educ Pract Ed 2007; 92 (3): ep87-91.

26.- Rodrigues AF, van Mourik ID, Sharif K, et al: Mana- gement of end-stage central venous access in children referred for possible small bowel transplantation. J Pediatr Gastroenterol Nutr 2006; 42 (4): 427-33.

27.- Alastair J, Millar $W$ : Non-transplant surgery for short bowel syndrome. Pediatr Surg Int 2013; 29: 983-7.

28.- King B, Carlson G, Khalil BA, Morabito A: Intestinal Bowel Lengthening in Children with Short Bowel Syndrome: Systematic Review of the Bianchi and STEP Procedures. World J Surg 2013; 37: 694-704.

29.- Sudan D: Advances in the nontransplant medical and surgical management of intestinal failure. Curr Opin Organ Transpl 2009; 14 (3): 274-9.

30.- Rege AS, Sudan DL: Autologous gastrointestinal reconstruction: review of the optimal nontransplant surgical options for adults and children with short bowel syndrome. Nutr Clin Pract 2013; 28 (1): 65-74.

31.- Fishbein TM: Intestinal Transplantation. N Engl J Med 2009; 361: 998-1008.

32.- Fishbein T, Matsumoto CS: Intestinal replacement therapy: Timing and Indications for referral of patients to an intestinal rehabilitation and transplant program. Gastroenterology 2006; 130: Suppl 1: S147-151.

33.- Beath S, Pironi L, Gabe $S$, et al: Collaborative strategies to reduce mortality and morbidity in patients with chronic intestinal failure including those who are reffered for small bowel transplantation. Transplantation 2008; 85 : 1378-84.

34.- Starzl TE, Todo S, Tzakis A, et al: The many faces of intestinal transplantation. Surg Gynecol Obstet 1991; 172: 335-44.

35.- Sudan DL, Chinnakotla S, Horslen S, et al: Basiliximab decreases the incidence of acute rejection after intestinal transplantation. Transplant Proc 2002; 34: 940-1.

36.- Fishbein TM, Florman S, Gondolesi G, et al: Intestinal transplantation before and after the introduction of sirolimus. Transplantation 2002;73: 1538-42.

37.- Primeggia J, Matsumoto CS, Fishbein TM, et al: Infections among adult small bowel and multivisceral transplant recipients in the 30 -day postoperative period. Transpl Inf Dis 2013; 0: 1-8.

38.- Abu-Elgmad K, Kosmach-Park B, Costa G, et al: Longterm survival, Nutrition autonomy and quality of life after Intestinal and Multivisceral Trasnsplantation. Ann Surg 2012; 256: 494-508.

39.- Girlanda R, Cheema AK, Kaur P, et al: Metabolomics of Human Intestinal Transplantation 2012; Supp14: S1826.

40.- Goulet $O$, Ruemmele F, Lacaille F, et al: Irreversible Intestinal Failure. J Pediatr Gastroenterol Nutr 2004; 38 (3): 250-69. 